\title{
Effect of Tongfu traditional Chinese medicine preparation on patients with septic gastrointestinal dysfunction: a systematic review and meta-analysis
}

\author{
Lijuan Zhang ${ }^{1,2}$, Qiaomei $\mathbf{W u}^{1,2}$, Peipei Chen ${ }^{1,2}$, Yan Xu ${ }^{1,2}$, Yafang Zheng ${ }^{1,2}$, Shihong Ma $^{1,2}$, Jingxia Zheng ${ }^{1,2}$ \\ ${ }^{1}$ The Second Affiliated Hospital of Guangzhou University of Chinese Medicine, Guangdong Provincial Hospital of Chinese Medicine, Guangzhou, \\ China; ${ }^{2}$ The Second Clinical College of Guangzhou University of Chinese Medicine, Guangzhou, China \\ Contributions: (I) Conception and design: L Zhang; (II) Administrative support: J Zheng; (III) Provision of study materials or patients: Q Wu, P Chen; \\ (IV) Collection and assembly of data: Y Xu, Y Zheng; (V) Data analysis and interpretation: J Zheng, S Ma; (VI) Manuscript writing: All authors; (VII) \\ Final approval of manuscript: All authors. \\ Correspondence to: Jingxia Zheng. The Second Affiliated Hospital of Guangzhou University of Chinese Medicine \& Guangdong Provincial Hospital of \\ Chinese Medicine, No 111, Dade Road, Yuexiu District, Guangzhou 510120, China. Email: 13622760199@126.com.
}

Background: Tongfu traditional Chinese medicine (TCM) preparation is a common alternative therapy
for clinical treatment of patients with septic gastrointestinal dysfunction. In recent years, a number of
randomized controlled trials (RCTs) have been conducted to verify the effectiveness of Tongfu TCM
preparation in the treatment of sepsis gastrointestinal dysfunction, but all of them have been small sample
studies, and the research conclusions have been controversial. Here, this study conducted a meta-analysis on
the clinical efficacy of the treatment of septic gastrointestinal dysfunction with TCM preparation, to produce
a more objective and comprehensive systematic review to guide clinical application.
To evaluate the efficacy and safety of Tongfu traditional Chinese medicine (TCM) preparation in the
treatment of patients with septic gastrointestinal dysfunction.

Methods: Randomized controlled trials (RCTs) of Tongfu TCM preparation in the treatment of septic gastrointestinal dysfunction published before February 2021 were searched for in the Chinese databases China National Knowledge Infrastructure (CNKI), Wanfang, Chinese Biomedical Literature Disk (CBMdisc), and Chongqing VIP (CQVIP), and English databases PubMed, Web of Science, EBSCO, and The Cochrane Library. Meta-analysis was performed using RevMan 5.3 software, and funnel plots were drawn to evaluate the bias of literatures.

Results: A total of 22 RCTs involving 1,558 patients were included. There were 772 patients in the control group and 786 in the trial group. Meta-analysis results showed that: gastrointestinal dysfunction score [mean difference $(\mathrm{MD})=-0.50,95 \%$ confidence interval (CI): -0.61 to -0.38 ], Acute Physiology and Chronic Health Evaluation II (APACHE II) score ( $\mathrm{MD}=-3.30,95 \% \mathrm{CI}:-3.73$ to -2.86 ), and mortality (MD =0.34, 95\% CI: $0.25-0.47)$ in the experimental group were significantly lower than those in the control group, and the difference was statistically significant $(\mathrm{P}<0.001)$. The funnel plot results showed that there was little possibility of publication bias.

Discussion: Tongfu TCM preparation can effectively improve the gastrointestinal function of patients with sepsis gastrointestinal dysfunction, prevent the deterioration of the disease, and reduce the mortality; however, more evidence is required to substantiate these findings.

Keywords: Tongfu; Chinese medicine preparation; sepsis; gastrointestinal dysfunction; meta-analysis

Submitted Jul 26, 2021. Accepted for publication Oct 14, 2021.

doi: 10.21037/apm-21-2461

View this article at: https://dx.doi.org/10.21037/apm-21-2461 


\section{Introduction}

Sepsis is a life-threatening impairment of organ function caused by the dysregulation of host response triggered by infection. In essence, it is a systemic inflammatory response of the body to various infectious factors. With the further development of sepsis, multiple organ dysfunction syndrome (MODS) may occur, which is one of the main causes of death in critically ill patients (1). According to statistics, about 31.5 million patients worldwide experience sepsis every year, and about 19.4 million patients manifest severe sepsis, among whom 5.3 million patients die (2). In recent years, despite some progress having been made in organ functional support technology and anti-infective treatment of sepsis, the mortality is still as high as $30-70 \%$, bringing a huge burden to the global medical and health system and social economy (3).

Gastrointestinal dysfunction is one of the common complications in patients with sepsis, and they are of mutual influence and causation. At present, it is generally believed that the gastrointestinal tract not only initiates the occurrence of sepsis, but is also a victim of sepsis. When sepsis occurs, the intestine is one of the most vulnerable organs, which can lead to gastrointestinal dysfunction, such as uncontrolled inflammation, ischemia and hypoxia, reperfusion injury, immunosuppression and intestinal flora disorders. At the same time, when gastrointestinal dysfunction occurs, its mucosal barrier is damaged. There are a large number of bacteria and endotoxins in the intestine that spread through the mucosal barrier to the tissues outside the intestine-bacteria translocate, which further damages various organs and causes a second blow, so a vicious circle is formed. In addition, intestinal infection caused by gastrointestinal injury can also induce sepsis, and if severe, it can lead to multiple organ dysfunction and even death $(4,5)$. Therefore, effective prevention and treatment of gastrointestinal dysfunction is one of the keys to reduce the mortality of patients with sepsis. Traditional Chinese medicine (TCM) holds that the function of the stomach and intestines can be maintained when the Qi descends unimpededly. If communication between the upper and lower Qi is obstructed, stasis of hollow organ (Fu)-Qi can lead to gastrointestinal dysfunction, such as abdominal distension, constipation, gastric retention, and even vomiting reflux and other manifestations. Symptoms of gastrointestinal dysfunction in sepsis are mostly caused by adverse rising of the stomach-Qi and obstruction of $\mathrm{Fu}-$ Qi. Based on the theoretical tenet that "the fluency of the six-Fu indicates its usability, the descending Qi reflects the fluency of the ascending and descending Qi pathways", the treatment principle is mainly to dredge the $\mathrm{Fu}(6)$. Among the traditional Chinese medicine preparations for intestinal tract, the main drug is rhubarb, which is aimed at dredging qi of Fu-organ and subsiding reverse function. At the same time, drugs such as strengthening the body's vitality, warming yang and benefiting qi, attacking blood stasis in the body, clearing away heat and detoxifying are added to prevent excessive diarrhea, which injures the body's stomach and causes the spleen and stomach to be cold. It is recommended to replenish the righteous qi and attack the pathogens at the same time, adjust the qi and restore it to a relatively coordinated state, so as to achieve the effect of gastrointestinal detoxification. A meta-analysis showed that traditional Chinese medicine preparations for intestinal tract can reduce the hospital mortality of ARDS patients, shorten the mechanical ventilation time and ICU hospital stay, and improve the respiratory mechanics parameters (7). However, we have not yet found a metaanalysis on the effect of general traditional Chinese medicine preparations on patients with sepsis and gastrointestinal dysfunction. In recent years, a number of randomized controlled trials (RCTs) have been conducted to verify the effectiveness of Tongfu TCM preparation in the treatment of sepsis gastrointestinal dysfunction, but all of them have been small sample studies, and the research conclusions have been controversial. In order to further clarify the effectiveness of Tongfu TCM preparation in the treatment of sepsis gastrointestinal dysfunction, this study conducted a systematic evaluation of published RCTs on Tongfu TCM preparation in the treatment of septic gastrointestinal dysfunction. This study provides a reference for the prospect and future research of Tongfu TCM preparation in the treatment of sepsis gastrointestinal dysfunction. We present the following article in accordance with the PRISMA reporting checklist (available at https:// dx.doi.org/10.21037/apm-21-2461).

\section{Methods}

\section{Inclusion and exclusion criteria}

The inclusion criteria were as follows: (I) research type: randomized controlled trial in English and Chinese; (II) participants: patients over 18 years of age with septic gastrointestinal dysfunction; (III) interventions: experimental group: Traditional Western medicine treatment combined with Tongfu TCM preparation, including Tongfu TCM decoction or preparation. Control 
group: Western medicine conventional treatment but not including TCM treatment; (IV) Outcome: Main outcome: Gastrointestinal dysfunction score. Secondary outcome: (i) Acute Physiology and Chronic Health Evaluation II (APACHE II) score; (ii) mortality.

The exclusion criteria were as follows: (I) case report, animal experiment, treatment experience, literature review, retrospective study; (II) patients with primary gastrointestinal diseases, such as primary gastrointestinal motility disorders, gastrointestinal bleeding, gastrointestinal tumors, or a history of gastrointestinal surgery; (III) republished literature; (IV) the full text was unable to be obtained; (V) the original research data could not be extracted or was missing.

\section{Literature retrieval strategy}

We searched for RCTs of Tongfu TCM preparation in the treatment of septic gastrointestinal dysfunction published before February 2021 in the Chinese databases China National Knowledge Infrastructure (CNKI), Wanfang, Chinese Biomedical Literature Disk (CBMdisc), and Chongqing VIP (CQVIP), and the English databases PubMed, Web of Science, EBSCO, and The Cochrane Library. At the same time, manual retrieval was carried out for references in the included literatures to maximize the obtainment of relevant literatures. The retrieval was carried out by combining Medical Subject Headings $(\mathrm{MeSH})$ with free words. Chinese database search terms included the following: tongfu, tongxia, xiexia, xiafa, enema, rhubarb, mirabilite, chengqi soup, traditional Chinese medicine, Chinese herbal medicine, sepsis, multiple organ dysfunction, multiple organ failure, acute gastrointestinal injury/gastrointestinal dysfunction, and so on. English database search words included: Tongfu, relax bowel, purgation, purgative, enema, rhubarb, mirabilite, dachengqi decoction, Chinese medicine, herbal, sepsis, MODS, acute gastrointestinal injury/gastrointestinal dysfunction, and so on.

\section{Literature screening and data extraction}

According to the inclusion and exclusion criteria, 2 researchers independently screened the literature, extracted the data, and filled in the information extraction form for cross-checking. If there was disagreement and it was unclear whether the literature should be included, resolution was sought through discussion or negotiation with a third researcher. During literature screening, the title and abstract were read first. If the inclusion criteria were met, the full text was read to further determine inclusion. In the case of lacking important information, attempts were made to contact the original study author to provide additional information. Data extraction content included: (I) basic information of literature: title, first author, publication year, journal, and so on; (II) baseline characteristics and intervention measures of participants; (III) the key elements of literature quality evaluation; (IV) outcome indicators and outcome measurement data.

\section{Literature quality assessment}

The quality of the literature was independently evaluated by 2 investigators using the assessment tool of RCT risk of bias recommended by the Cochrane Manual, Version 5.3.0. After completion of the evaluation, the 2 researchers discussed and reached a consensus on the literature quality evaluation results. In case of disagreement, the 2 researchers engaged in discussion or negotiated with a third researcher to reach a consensus.

\section{Statistical analysis}

The software Rev Man 5.3 (ReviewManager 5.3; Copenhagen: The Nordic Cochrane Center, The Cochrane Collaborative, 2014) was used for meta-analysis. Weighted number difference (MD) OR standard mean difference (SMD) were used as effect indexes for measurement data, and relative risk (RR) or odds ratio (OR) were used as effect indexes for enumeration data. Point estimates and 95\% confidence interval (CI) were given for each effect size. The chi-square $\left(\chi^{2}\right)$ test was used to determine whether there was statistical heterogeneity among the included study results, and $\mathrm{I}^{2}$ test was used to determine the magnitude of heterogeneity. If $\mathrm{P}>0.1$ and $\mathrm{I}^{2}<50 \%$, no statistical heterogeneity was considered, and a fixed-effect model was selected for meta-analysis. If $\mathrm{P}<0.1$ and $\mathrm{I}^{2}>50 \%$, statistical heterogeneity was considered, and further analysis of the source of heterogeneity was needed. After excluding the influence of obvious clinical heterogeneity, a randomeffects model was selected for meta-analysis. When the clinical heterogeneity between the 2 studies is large, only descriptive analysis will be undertaken. To evaluate the effects of different observation time on pooled estimates, we performed a subgroup analysis stratified by observation time for outcomes with enough included studies. In this 
work, a sensitivity analysis will be performed to access the robustness of the results. In this work, we will conduct sensitivity analysis on gastrointestinal dysfunction score, APACHE II score, and mortality by changing different effect models and statistical methods. The difference between the re-obtained effects and the original effects will be compared. If the results of the Meta analysis cannot be substantially changed, it indicates that the stability and reliability of the Meta analysis are relatively good. When the number of included studies was $\geq 10$, funnel plots were used to determine whether there was publication bias. A P value $<0.05$ was considered statistically significant.

\section{Results}

\section{Literature retrieval results}

A preliminary examination of 815 relevant literatures was conducted, including 778 studies in Chinese and 37 in English. After deletion of duplicate literatures by NoteExpress, 417 studies remained. According to the screening criteria, we read the titles and abstracts, which led to the exclusion of 311 literatures due them being reviews, animal experiments, and inconsistent with the topic, and initially included 106 literatures. After rescreening by reading the full text, 84 articles that did not meet the inclusion criteria were excluded, including repeated publication, non-RCTs, non-sepsis gastrointestinal dysfunction, non-Tongfu-type TCM preparations or Tongfu-type TCM preparations combined with other TCM treatment measures. A total of 22 articles were finally included. The flow chart of literature screening is shown in Figure 1.

\section{The basic characteristics of the included studies and the results of bias risk assessment}

The basic characteristics of the included studies are shown in Table 1. The results of bias risk assessment are shown in Table 2.

\section{Meta-analysis results}

\section{Gastrointestinal dysfunction score}

Among the included literatures, 15 reported the effects of Tongfu TCM preparations on the scores of gastrointestinal dysfunctions in patients with sepsis (8-11,13-16,21-23,25-27,29). A total of 1,214 participants were included, including 616 in the experimental group and 598 in the control group. The results of meta-analysis showed that the score of gastrointestinal dysfunction in the experimental group was significantly lower than that in the control group (random-effects model, $\mathrm{MD}=-0.50,95 \% \mathrm{CI}$ : -0.61 to $-0.38, \mathrm{P}<0.001)$. Subgroup analysis was conducted according to different observation time, and the results showed that no matter whether the intervention lasted for 3,7 , or 14 days, the score of gastrointestinal dysfunction in the experimental group was significantly lower than that in the control group, with statistically significant differences (Figure 2).

\section{APACHE II score}

Among the included literatures, 15 reported the effects of Tongfu TCM preparations on APACHE II score of patients with septic gastrointestinal dysfunction (9-16,21-23,25-27,29). A total of 1,461 participants were included, including 740 in the experimental group and 721 in the control group. The results of meta-analysis showed that the APACHE II score of the experimental group was significantly lower than that of the control group, and the difference was statistically significant (fixed effect model, $\mathrm{MD}=-3.30,95 \% \mathrm{CI}:-3.73$ to $-2.86, \mathrm{P}<0.001)$. Subgroup analysis was conducted according to different observation times, and the results showed that no matter whether the intervention lasted for 3, 5, 7, or 14 days, the APACHE II score of the experimental group was significantly lower than that of the control group, with statistically significant differences (Figure 3).

\section{Mortality}

Among the included literatures, 12 reported the effect of Tongfu TCM preparations on the mortality rate of patients with septic gastrointestinal dysfunction $(10,11,13,16,18-22,24,27,29)$. A total of 997 participants were included, including 507 in the experimental group and 490 in the control group. The results of meta-analysis showed that the mortality of the experimental group was significantly lower than that of the control group, and the difference was statistically significant (fixed effect model, $\mathrm{MD}=0.34,95 \%$ CI: $0.25-0.47, \mathrm{P}<0.001)$. Subgroup analysis was conducted according to different observation time, and the results showed that the mortality of the experimental group was significantly lower than that of the control group at 14 or 28 days after intervention, with statistically significant differences (Figure 4). 


\section{Identification of studies via databases and registers}
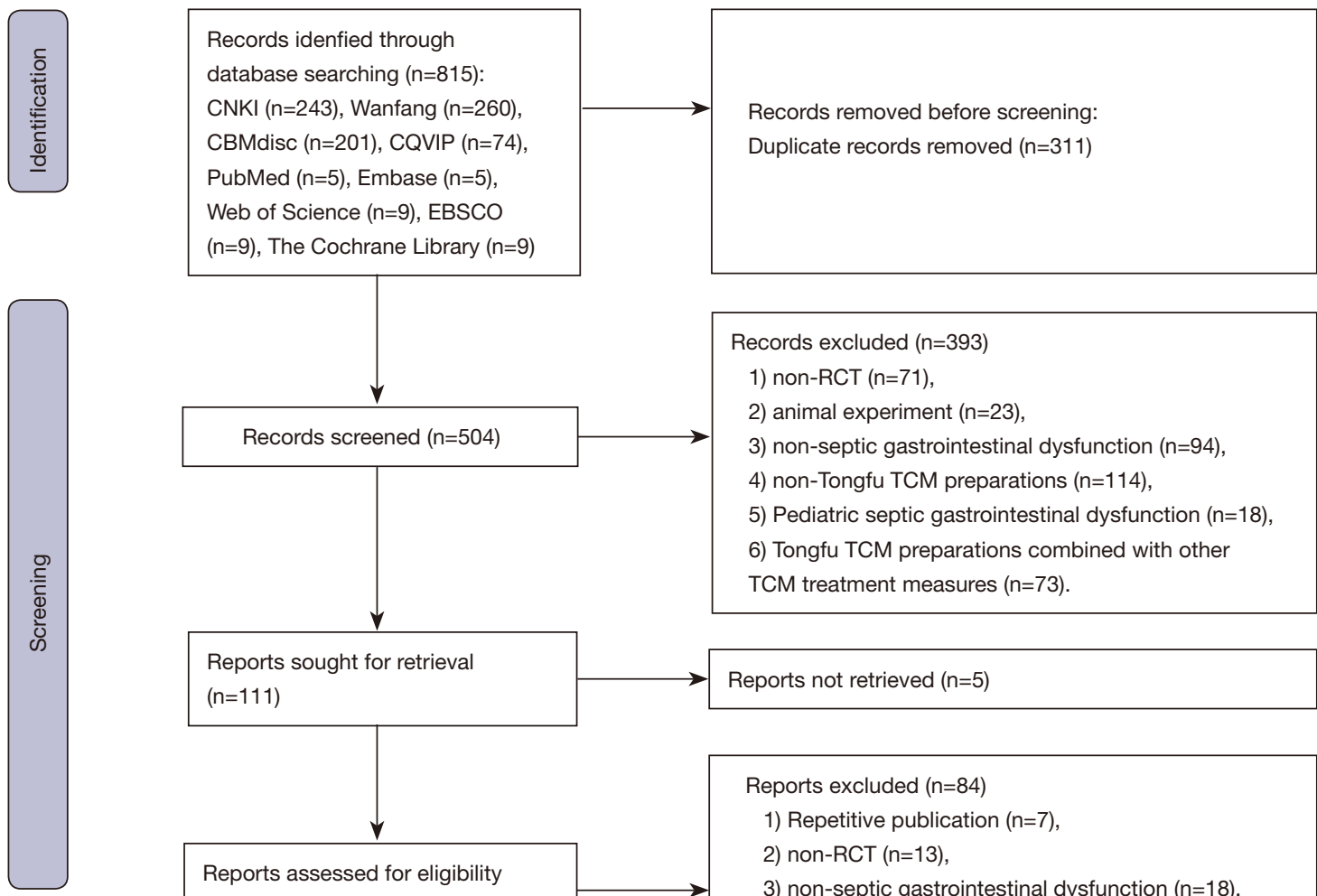

Records excluded $(n=393)$

1) non-RCT $(n=71)$,

2) animal experiment $(n=23)$,

3) non-septic gastrointestinal dysfunction $(n=94)$,

4) non-Tongfu TCM preparations $(n=114)$,

5) Pediatric septic gastrointestinal dysfunction $(n=18)$,

6) Tongfu TCM preparations combined with other

TCM treatment measures $(n=73)$.

Reports not retrieved $(n=5)$

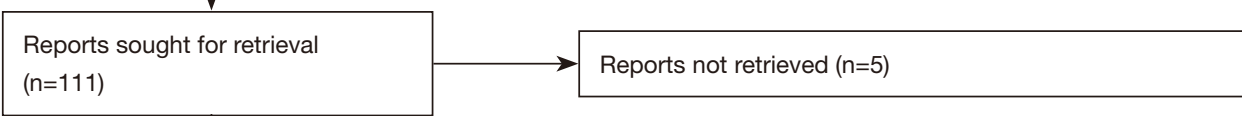

Reports excluded ( $\mathrm{n}=84)$

1) Repetitive publication $(n=7)$,

2) non-RCT $(n=13)$,

3) non-septic gastrointestinal dysfunction $(n=18)$,

4) non-Tongfu TCM preparations $(n=14)$,

5) Tongfu TCM preparations combined with other

TCM treatment measures $(n=20)$,

6) low quality $(n=9)$,

7) other $(n=3)$.

Studies included in review

$(\mathrm{n}=22)$

Reports of included studies

$(\mathrm{n}=22)$

Figure 1 The flow chart of literature screening and results. RCT, randomized controlled trial; TCM, traditional Chinese medicine.

\section{Sensitivity analysis and publication bias}

\section{Sensitivity analysis}

The sensitivity analysis of gastrointestinal dysfunction score, APACHE II score, and mortality was carried out using the transformation effect model and different statistical methods. The results of the meta-analysis showed that the OR value, $95 \% \mathrm{CI}$, and $\mathrm{P}$ value were very similar before and after the sensitivity analysis, except for the statistical method before and after the APACHE II score conversion. Other results did not change significantly, suggesting that the stability and reliability of the results were relatively good (Table 3).

\section{Publication bias}

Outcome indicators of $\geq 10$ included literatures, such as gastrointestinal dysfunction score, APACHE II score, and case fatality rate, were evaluated by funnel plots. The funnel plot was inverted and fundamental symmetrical, and the distribution of each study site was relatively uniform, suggesting that there was little possibility of publication bias (Figures 5-7). 


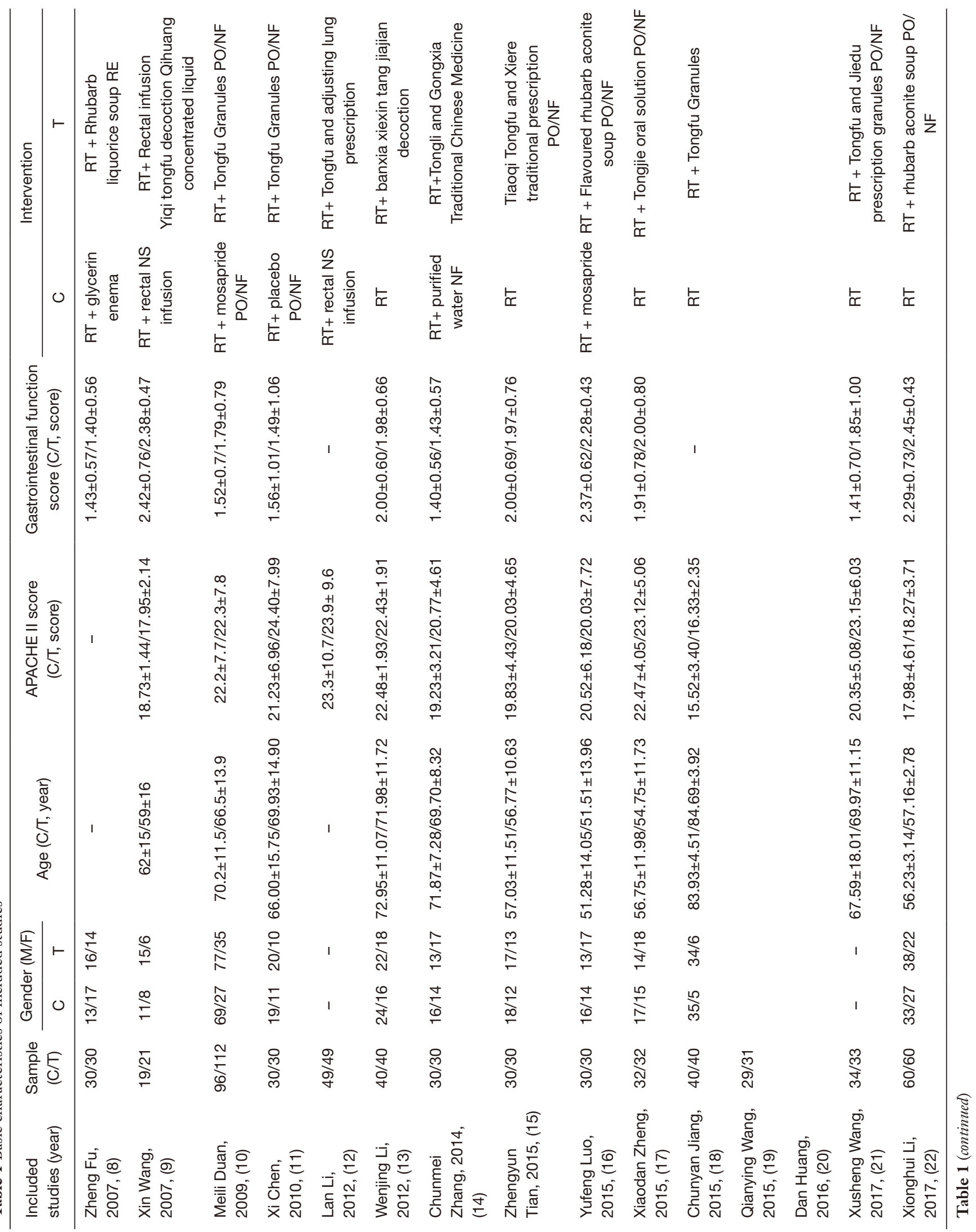




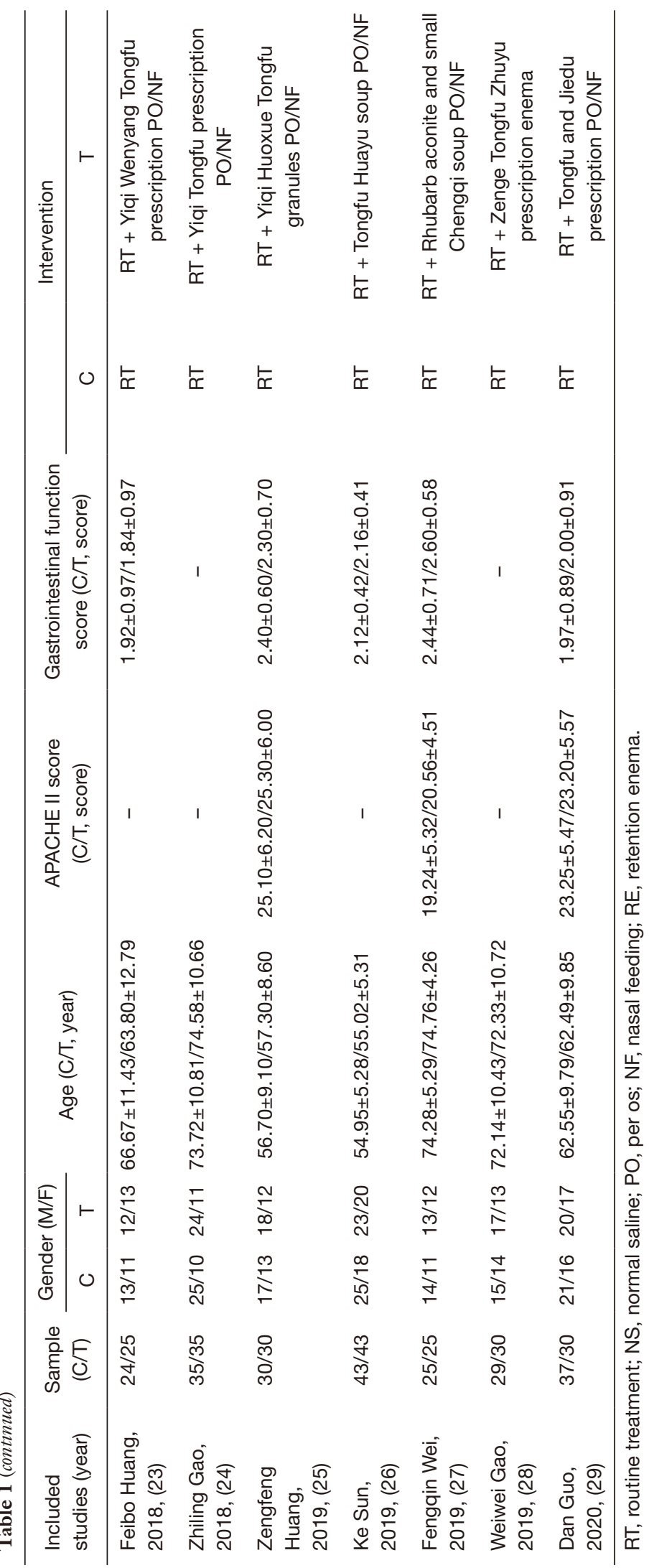


Table 2 Risk of bias assessment for included studies

\begin{tabular}{|c|c|c|c|c|c|c|}
\hline Included studies (year) & Random method & $\begin{array}{c}\text { Allocation } \\
\text { concealment }\end{array}$ & $\begin{array}{l}\text { Blinding } \\
\text { method }\end{array}$ & $\begin{array}{l}\text { The integrity of the data } \\
\text { (exit/lost to follow-up) }\end{array}$ & $\begin{array}{l}\text { Selectively report } \\
\text { research results }\end{array}$ & $\begin{array}{c}\text { Other sources } \\
\text { of bias }\end{array}$ \\
\hline Zheng Fu, 2007, (8) & Unclear & Unclear & No & Unclear & Unclear & Unclear \\
\hline Xin Wang, 2007, (9) & Unclear & Unclear & No & Unclear & Unclear & Unclear \\
\hline Meili Duan, 2009, (10) & BRE & Unclear & No & Unclear & Unclear & Unclear \\
\hline Lan Li, 2012, (12) & RNT & Unclear & No & Unclear & Unclear & Unclear \\
\hline Wenjing Li, 2012, (13) & Unclear & Unclear & Single blind & Unclear & Unclear & Unclear \\
\hline Chunmei Zhang, 2014, (14) & Computer & Unclear & No & Unclear & Unclear & Unclear \\
\hline Zhengyun Tian, 2015, (15) & RNT & Unclear & No & Unclear & Unclear & Unclear \\
\hline Qianying Wang, 2015, (19) & RNT & Unclear & No & Unclear & Unclear & Unclear \\
\hline Dan Huang, 2016, (20) & RNT & Unclear & Single blind & Unclear & Unclear & Unclear \\
\hline Xusheng Wang, 2017, (21) & RNT & Unclear & No & Unclear & Unclear & Unclear \\
\hline Xionghui Li, 2017, (22) & Unclear & Unclear & No & Unclear & Unclear & Unclear \\
\hline Feibo Huang, 2018, (23) & RNT & Unclear & No & Unclear & Unclear & Unclear \\
\hline Zhiling Gao, 2018, (24) & $\begin{array}{c}\text { SAS } \\
\text { programming }\end{array}$ & $\begin{array}{l}\text { An opaque } \\
\text { envelope }\end{array}$ & No & Unclear & Unclear & Unclear \\
\hline
\end{tabular}

BRE, block random envelope method; RNT, random number table; CTM, coin toss method.

\section{Discussion}

\section{Methodological quality of the included literatures}

This study systematically analyzed RCTs on the effects of Tongfu TCM preparations on patients with septic gastrointestinal dysfunction. The literature quality was evaluated according to the RCT bias risk assessment tool recommended by the Cochrane Manual Version 5.3.0, which revealed that the overall literature quality needed to be improved. Prominent problems in research design included: in the generation of random assignment sequence, most of the studies did not give specific random methods; allocation scheme concealment was ignored in all study designs, which could lead to selective bias; no studies had truly achieved the double-blind method between participants and interventionists, which may have resulted in measurement bias; most of the studies did not directly describe the situation of withdrawal or loss of follow-up, which may have led to loss of follow-up bias; most of the studies did not report the relevant information of adverse reactions, so safety analysis could not be conducted; the result report was not standardized; all the included studies were Chinese studies, which affected the generalizability of the analyzed results. These problems exist widely in TCM clinical studies, which affect the reproducibility and authenticity of the conclusions to some extent, and have 


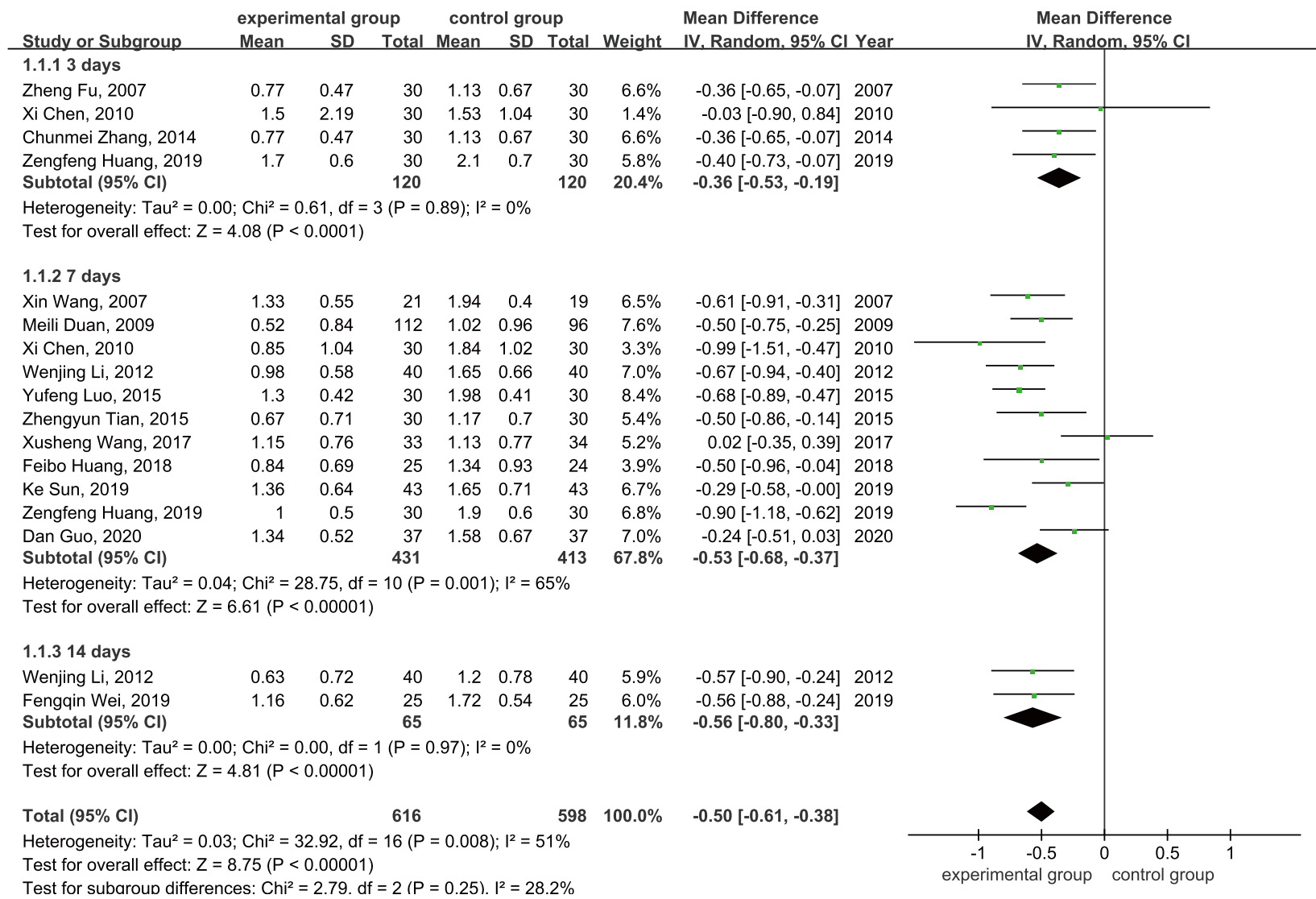

Figure 2 Influence of Tongfu TCM preparation on gastrointestinal dysfunction scores of patients with sepsis. TCM, traditional Chinese medicine; CI, confidence interval; SD, standard deviation.

been highlighted by international evidence-based medicine academics.

\section{Effect of Tongfu TCM preparation on patients with septic gastrointestinal dysfunction}

Pathogenic toxin can cause reverse and disordered qi movement in patients with sepsis; as a result, the spleen loses its ability in ascending clear, the stomach loses its ability in descending turbidity, and the lungs lose their ability in purification and descending. The lungs and the large intestine are both superficies and interior for each other. The lungs are zang-viscera, and the intestine is a fu-viscera; they are both superficies and interior for each other and can affect each other. When the lung qi descends, the transportation function of the intestines is normal; when the lung qi is reversed and ascends, the intestine experiences qi constipation and excessive heat, leading to the accumulation of pathogenic toxin, which will turn into heat to consume and injure fluids; finally, the intestine loses its nutrition and the fu-viscera will be blocked, resulting in gastrointestinal dysfunction. The clinical manifestations of gastrointestinal dysfunction include abdominal distension, constipation, and little or no gas passing, which are quite similar to the distention, fullness, dryness, and excess of the syndrome of excess of Yangming fu-viscera in TCM (30). According to TCM, the six fu-viscera mainly function to transport and transform food and water, with urethral orifice and anus being the main channels for discharging turbid pathogen from human body. If the lung qi loses its function in purification and descending, it will be difficult to promote the discharge of turbid pathogen from the intestinal tract; accordingly, a large number of pathogenic toxin will gather in the intestinal tract, damage the intestinal tract, and make the latter become a new pathway for the invasion of pathogenic toxin. From the perspective of Western medicine, such a condition increases the abnormal reproduction of intestinal bacteria and the 


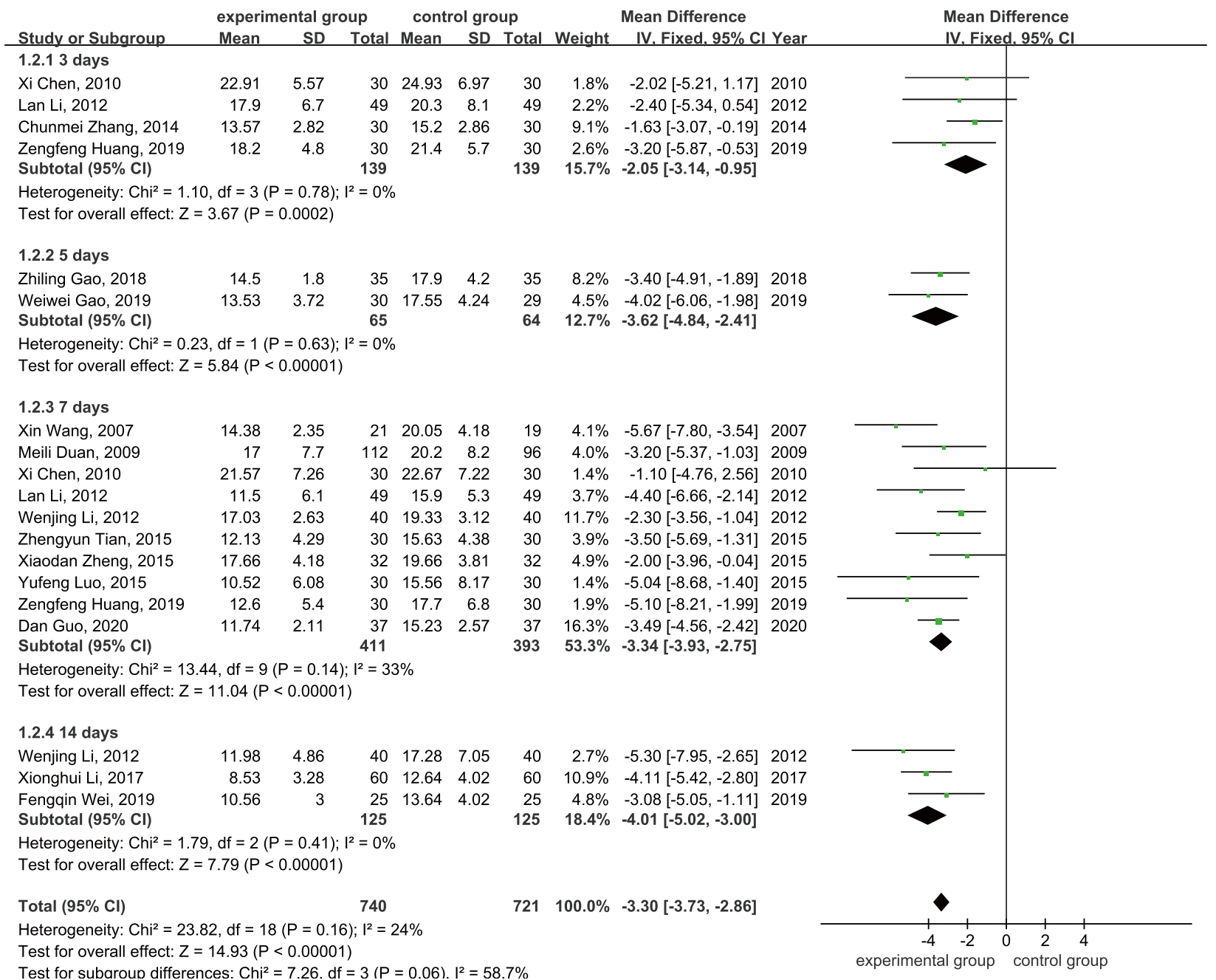

Figure 3 Influence of tongfu TCM preparation on APACHE II score of patients with septic gastrointestinal dysfunction. TCM, traditional Chinese medicine; CI, confidence interval; SD, standard deviation.

harmful effects of toxoids on the body, resulting in various complications or aggravating the primary disease (31). In TCM hospitals or clinics, Tongfu TCM preparations are often used after syndrome differentiation. Tongfu means dredging the qi in fu-viscera, regulating the visceral qi movement, and regulating the ascending and descending functions of the spleen and stomach, which corresponds to promoting gastrointestinal motor function, discharging toxic and harmful products, breaking the vicious cycle caused by toxin retention, improving intestinal microcirculation, reducing ischemia-reperfusion injury, regulating the internal environment of the human body, and thus preventing the worsening of the disease in modern medicine (32). Many studies $(25,26,28)$ have demonstrated that Tongfu TCM preparations, whenever administered orally, rectally, or by nasal feeding, can improve gastrointestinal dysfunction and reduce case-fatality rate in appropriately selected patients.

In our current Meta analysis, Tongfu TCM preparations were compared with conventional care in septic patients who have received routine treatment and nursing. The results showed that the gastrointestinal dysfunction score (MD $=-0.50,95 \%$ CI: -0.61 to -0.38$)$, APACHE II score $(\mathrm{MD}=-3.30,95 \% \mathrm{CI}:-3.73$ to -2.86$)$, and mortality rate (MD $=0.34,95 \%$ CI: $0.25-0.47)$ of the Tongfu TCM preparations group were significantly lower than those of the control group (all $\mathrm{P}<0.001$ ), suggesting that Tongfu TCM preparations can improve gastrointestinal function, prevent disease deterioration, and reduce mortality in 


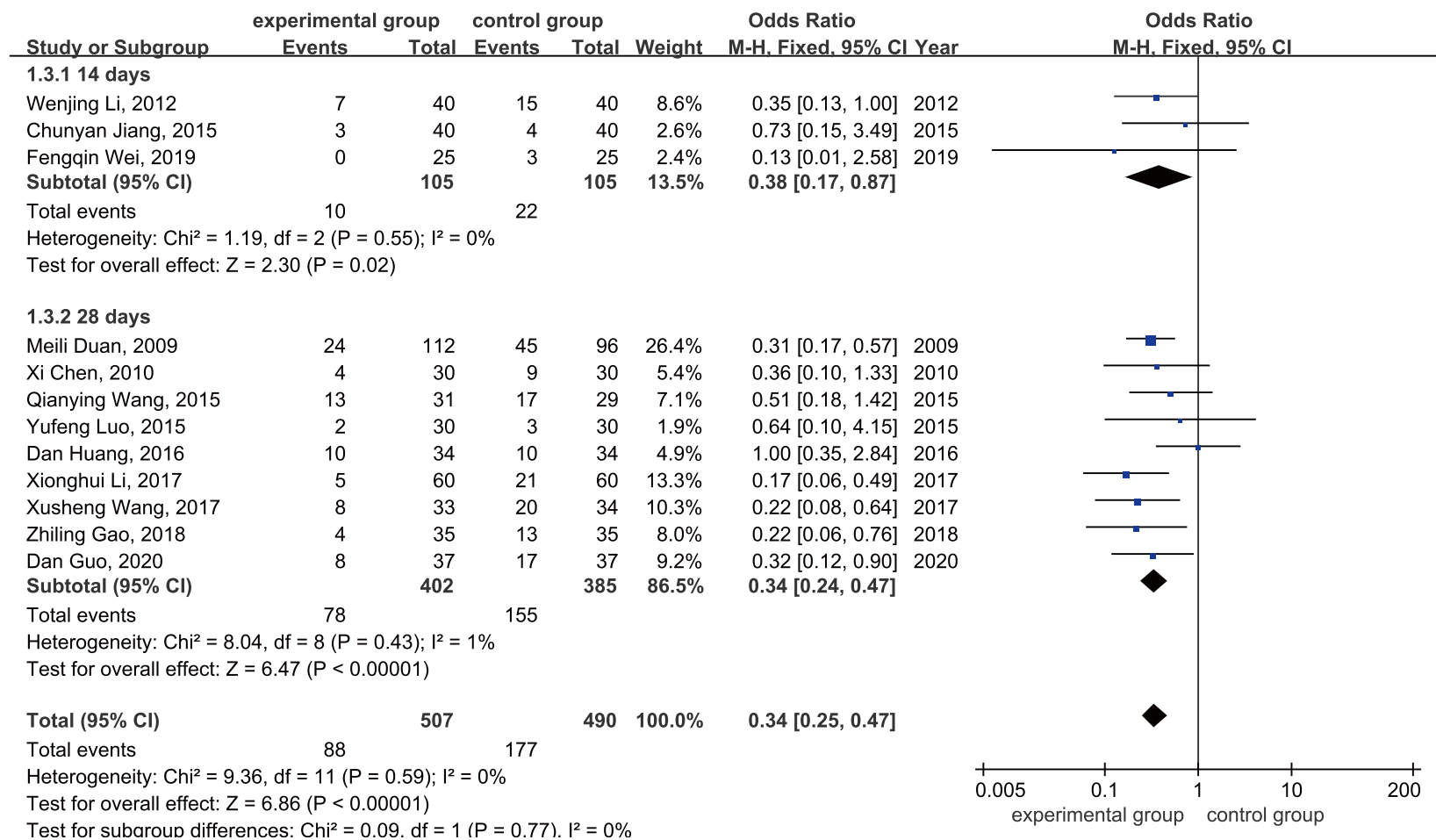

Figure 4 The effect of Tongfu TCM preparation on the mortality rate of patients with sepsis gastrointestinal dysfunction. TCM, traditional Chinese medicine; CI, confidence interval; SD, standard deviation.

Table 3 Sensitivity analysis of included references in the meta-analysis

\begin{tabular}{|c|c|c|c|c|}
\hline Effect indicators & \multicolumn{2}{|c|}{ Statistical pattern } & \multicolumn{2}{|c|}{ Effect model } \\
\hline $\begin{array}{l}\text { Gastrointestinal } \\
\text { dysfunction score }\end{array}$ & $\begin{array}{c}\text { RE: MD }(95 \% \mathrm{Cl}): \\
-0.50(-0.61 \text { to }-0.38)\end{array}$ & $\begin{array}{l}\text { RE: SMD (95\% Cl): } \\
-0.72(-0.92 \text { to }-0.52)\end{array}$ & $\begin{array}{c}\text { RE: MD (95\% Cl): } \\
-0.50(-0.61 \text { to }-0.38)\end{array}$ & $\begin{array}{c}\text { FE: MD (95\% Cl): } \\
-0.51(-0.58 \text { to }-0.43)\end{array}$ \\
\hline APACHE II score & $\begin{array}{c}\text { FE: MD }(95 \% \mathrm{Cl}): \\
-3.30(-3.73 \text { to }-2.86)\end{array}$ & $\begin{array}{l}\text { FE: SMD }(95 \% \mathrm{Cl}) \text { : } \\
-0.71(-0.82 \text { to }-0.60)\end{array}$ & $\begin{array}{c}\text { RE: } \mathrm{MD}(95 \% \mathrm{Cl}): \\
-3.33(-3.86 \text { to }-2.81)\end{array}$ & $\begin{array}{c}\text { FE: MD }(95 \% \mathrm{Cl}): \\
-3.30(-3.73 \text { to }-2.86)\end{array}$ \\
\hline
\end{tabular}

$\mathrm{SMD}$, standard mean difference; $\mathrm{Cl}$, confidence interval; SD, standard deviation; RR, relative risk; OR, odds ratio; RE, random effect; FE, fixed effect; APACHE II, Acute Physiology and Chronic Health Evaluation II.

patients with sepsis. This is consistent with the results of Zhang's research (14). It is possible that traditional Chinese medicine preparations for intestinal tract can help increase gastrointestinal motility, protect the intestinal mucosal barrier, prevent the retention and reproduction of bacteria or reduce the secretion of endotoxins, inhibit the occurrence of sepsis, and prevent gastrointestinal dysfunction from further developing into MODS, thereby reducing the fatality rate of patients with sepsis. It can be seen that protecting the intestines is very important for improving the prognosis of patients with sepsis and increasing the success rate of treatment. This discovery is of great significance to the prevention and treatment of sepsis.

In the 22 articles included in this analysis, the Tongfu TCM preparations mentioned were mainly: (I) Tongfu and Fuzheng (i.e., strengthening vital qi) prescriptions; (II) preparations for supplementing qi and warming yang; (III) preparations for regulating qi and relieving heat; 


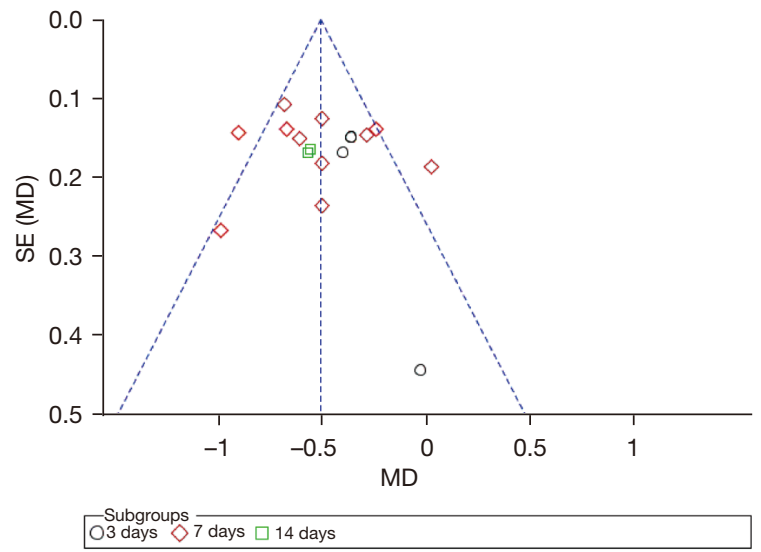

Figure 5 Funnel plot of gastrointestinal dysfunction score.

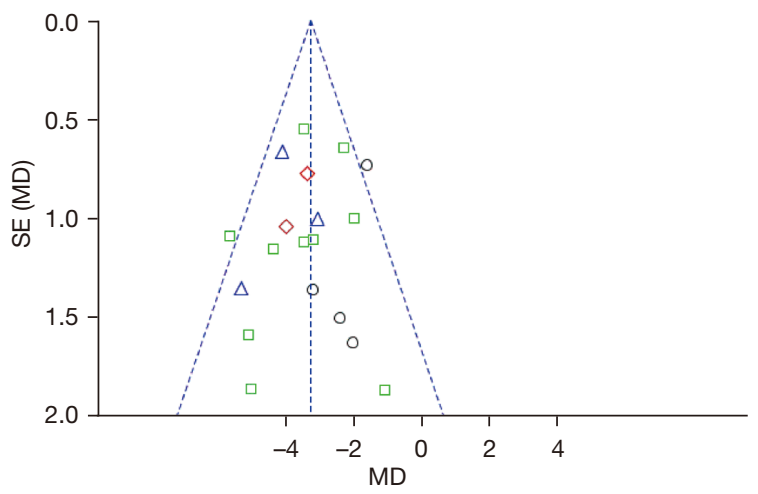

Subgroups
3 days $\triangle 5$ days $\square 7$ days $\triangle 14$ days

Figure 6 Funnel plot of APACHE II score.

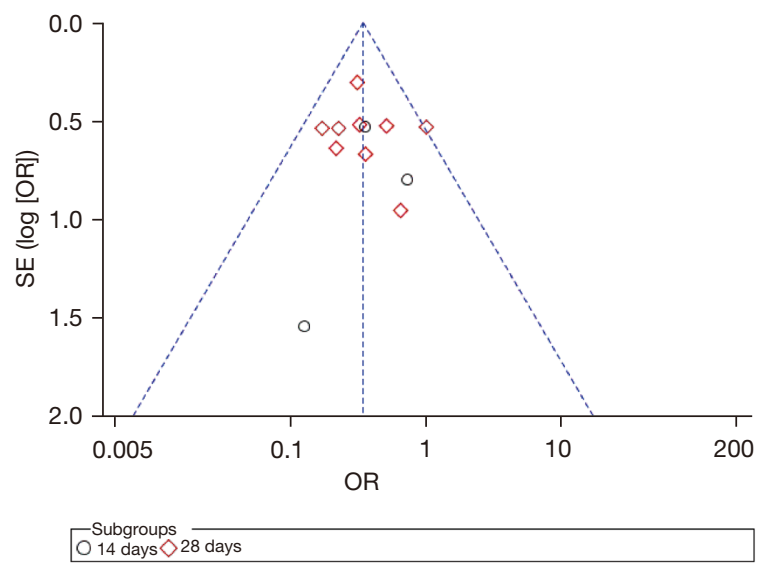

Figure 7 Funnel plot of mortality. or (IV) preparations for promoting blood circulation, removing blood stasis, and detoxification. Rhubarb root and rhizome was the most commonly used medicine in these preparations (in up to 21 articles). Literature (33) has shown that rhubarb root and rhizome can protect the barrier function of intestinal mucosa and has a definite efficacy in treating gastrointestinal dysfunction in patients with sepsis. Therefore, rhubarb root and rhizome is widely used in clinical settings. However, rhubarb root and rhizome is a purgative and has certain toxicity, and it should be used with caution in patients with chronic diseases. Unfortunately, few articles included in our current analysis described the adverse reactions and drug safety, which should be addressed in future research.

\section{Limitations of the study}

There were still some limitations in this paper: (I) the high literature bias may have affected the evidence quality of the systematic evaluation. (II) All the included studies were published in Chinese, and the study sites were all in China. The lack of relevant data from international studies limits the generalizability of the results. (III) Most of the included studies had small sample sizes and low methodological quality, and there was a possibility of selective bias and measurement bias. In addition, only 1 study reported the concealment of random allocation scheme, so it was impossible to judge whether it had genuinely followed the RCT research method. (IV) In the literature collected in this article, there are some differences in the traditional Chinese medicine preparations for intestinal tract formulas, and their components are quite different, but the theory of traditional Chinese medicine is the same. We have not considered thoroughly in the research process, and will pay attention to and perfect the research in future.

In conclusion, Tongfu TCM preparations can effectively improve the gastrointestinal function of patients with septic gastrointestinal dysfunction, prevent the deterioration of the disease, and reduce the mortality, but there was not enough evidence to strongly support these findings. It was suggested that future clinical trials should be carried out according to the standard of RCTs and reported according to the Consolidated Standards of Reporting Trials (CONSORT) declaration, especially the generation of random sequences and the implementation of the allocation concealment 
scheme. At the same time, it was recommended to use objective evaluation indicators as far as possible, to avoid the use of comprehensive indicators. We anticipate to more local and regional studies with large sample sizes to effectively control possible bias and improve the quality of evidence in related studies to better guide clinical decisionmaking.

\section{Acknowledgments}

Funding: This work is financed by grants from Guangdong Hospital of Traditional Chinese Medicine Science and Technology Research Projects (YN2019HL03), Guangdong Provincial Key Laboratory of Research on Emergency in TCM (2017B030314176) and Guangzhou Civil Affairs Bureau Planned Project of Science and Technology (2021MZK36).

\section{Footnote}

Reporting Checklist: The authors have completed the PRISMA reporting checklist. Available at https://dx.doi. org/10.21037/apm-21-2461

Conflicts of Interest: All authors have completed the ICMJE uniform disclosure form (available at https://dx.doi. org/10.21037/apm-21-2461). The authors have no conflicts of interest to declare.

Ethical Statement: The authors are accountable for all aspects of the work in ensuring that questions related to the accuracy or integrity of any part of the work are appropriately investigated and resolved.

Open Access Statement: This is an Open Access article distributed in accordance with the Creative Commons Attribution-NonCommercial-NoDerivs 4.0 International License (CC BY-NC-ND 4.0), which permits the noncommercial replication and distribution of the article with the strict proviso that no changes or edits are made and the original work is properly cited (including links to both the formal publication through the relevant DOI and the license). See: https://creativecommons.org/licenses/by-nc-nd/4.0/.

\section{References}

1. He X, Li D, Qiao L, et al. Progress of epidemiology and outcome for sepsis. Zhonghua Wei Zhong Bing Ji Jiu Yi
Xue 2018;30:486-9.

2. He X, Li D, Qiao L, et al. Advances in the epidemiology and prognosis of sepsis. Zhonghua Wei Zhong Bing Ji Jiu Yi Xue 2018;30:486-9.

3. $\mathrm{Wu} \mathrm{W}$, Fan $\mathrm{Z}$, Yao $\mathrm{C}$, et al. Platelet maximum aggregation rate serves as a marker in di-agnosis and prognosis in patients with sepsis. Ann Palliat Med 2020;9:847-57.

4. Liu DW. Critical care medicine: from bench to bedside by scoping sepsis 3.0. Chinese Journal of Practical Internal Medicine 2018;38:981-3.

5. Mittal R, Coopersmith CM. Redefining the gut as the motor of critical illness. Trends Mol Med 2014;20:214-23.

6. Zhang JL, Zeng QY. The relationship between sepsis and gastrointestinal function injury and its research progress. The Journal of Practical Medicine 2016;32:2925-7.

7. Cheng L, Zhang Y, He SY, et al. A systematic review and Meta-analysis of Tongfu Xiefei method in the treatment of acute respiratory distress syndrome. Chinese Critical Care Medicine 2020;32:970-5.

8. Fu Z. Observation on the effect of retention enema of rhubarb liquorice decoction on gastrointestinal dysfunction in patients with SIRS. Beijing: China Academy of Chinese Medical Science, 2007.

9. Wang X, Li M, Jia HR, et al. Clinical Research on Intervention in the functional Impairment of Stomach Intestine of MODS by Rectum Infusion. Beijing Journal of Traditional Chinese Medicine 2007;26:391-3.

10. Duan ML, Dong J, Chen X, et al. Effects of Integrated Chinese-Western Medicine on Gastrointestinal Dysfunction in 208 Patients with Multiple Organ Dysfunction Syndrome. Chinese Journal of Integrated Traditional and Western Medicine in Intensive and Critical Care 2009;16:30-3.

11. Chen X, Li A, Zhang SW, et al. Effects of Tongfu Granule on Intestinal Dysfunction in Patients with Multiple Organ Dysfunction Syndrome. Chinese Journal of Integrated Traditional And Western Medicine 2010;30:810-3.

12. Li L, Chen L, Huang RF, et al. A Clinical Research on Regulation of Gastrointestinal Function by Rectal Instillation of Tongfu Lifei Soup in Patients with Severe Sepsis or Multiple Organ Dysfunction Syndrome. Chinese Journal of Integrated Traditional and Western Medicine in Intensive and Critical Care 2012;19:209-12.

13. Li WJ. Clinical study on Adjust Qi and Stomach Method in Treating Sepsis with Gastrointestinal Dysfunction of Qijiyongzhi Type. Jinan: Shandong University of Traditional Chinese Medicine, 2012.

14. Zhang CM. Clinical Research on Gastrointestinal 
Dysfunction in Sepsis Treated with Tongligongxia Chinese Herb. Tianjin: Tianjin Medical University, 2014.

15. Tian ZY, Zhao H, Kong L, et al. Clinical Research on Regulating Gas, Purgation and Purging Heat in the Treatment of Sepsis with Gastrointestinal Dysfunction. Journal of Emergency in Traditional Chinese Medicine 2015;24:1528-9.

16. Luo YF. The Clinical Research of Wen Tong Branch in the Treatment of Sepsis Yang Relieving Empirical Gastrointestinal Dysfunction. Guangzhou: Guangzhou University of TCM, 2015.

17. Zheng XD. Clinical and Experimental Study on Preventing and Treating Acute Gastrointestinal Injury with Multiple Organ Dysfunction Syndrome by Tongyujiedu. Nanjing: Nanjing University of Traditional Chinese Medicine, 2015.

18. Jiang CY, Xu YX, Hao RR, et al. Effect of Compound Tongfu Granule on Intestinal Permeability in Elderly Sepsis Patients. Chinese Journal of Integrated Traditional and Western Medicine 2015;35:787-91.

19. Wang QY. Clinical Research on Purgative Therapy for Patients with AGI. Chengdu: Chengdu University of TCM, 2015.

20. Huang D, Zhang XZ, Yu CB, et al. A Clinical Study on Effects of Early Using Dahuang Fuzi Decoction to Improve Gastrointestinal Dysfunction in Patients with Sepsis. Chinese Journal of Integrated Traditional and Western Medicine in Intensive and Critical Care 2016;23:393-8.

21. Wang XS, Fan WJ, Wu ZS, et al. Clinical Research of Tongfu Jiedu Prescription on Improving the Gastrointestinal Score and the Short-term Prognosis of the Severe Sepsis. Chinese Journal of Basic Medicine in Traditional Chinese Medicine 2017;23:512-3.

22. Li XH, Zhang DS. Clinical Study of Dahuang-fuzi Decoction on Gastrointestinal Dysfunction in Sepsis Patients. Hebei Journal of Traditional Chinese Medicine 2017;39:356-60.

23. Huang FB, Xu JL, Wang FM, et al. Yiqi Wenyang Tongfu Method in Treatment of Multiple Organ Dysfunction Syndrome Complicated with Gastrointestinal Dysfunction. Chinese Archives of Traditional Chinese Medicine 2018;36:1238-41.

24. Gao ZL, Jin K, Zhao Y, et al. Clinical study of Yiqi Tongfu Decoction on the intervention of acute gastrointestinal injury in senile patients with severe sepsis. Lishizhen Medicine and Materia Medica Research 2018;29:902-4.

25. Huang ZF, Chen RK, Fang C, et al. Protective effect of yiqi huoxue tongfu granules on intestinal mechanical barrier in patients with sepsis gastrointestinal dysfunction. Zhejiang Journal of Traditional Chinese Medicine 2019;54:94-5.

26. Sun Ke, Xia Guolian, Chen Zheqi. Effect of tongfu huayu decoction on gastrointestinal dysfunction in patients with sepsis. Chinese Traditional Chinese Medicine Science and Technology 2019;26:547-9.

27. Wei FQ, Lv J, Meng H, et al. Effect of Dahuang Fuzi Decoction Combined with Xiaochengqi Decoction on Gastrointestinal Function and Inflammatory Factors in Patients with Sepsis. Journal of Anhui University of Chinese Medicine 2019;38:36-41.

28. Gao WW, Kan JY, Yu NH. Effect of Zengye Tongfu Zhuyu Decoction Enema on Intestinal Barrier Repair in Patients with Sepsis Acute Gastrointestinal Injury. Tianjin Journal of traditional Chinese Medicine 2019;36:963-66.

29. Guo D, Yao XM, Li HJ. Study on Effect of Tongfu Jiedu Recipe on Gastrointestinal Dysfunction and Changes of DAO and PCT in Patients with Sepsis. Chinese Archives of Traditional Chinese Medicine 2020;39:86-8.

30. Lou $\mathrm{Y}, \mathrm{Wu} \mathrm{YW}, \mathrm{Xu} \mathrm{ZB}$. Research advances in traditional Chinese medicine in treating septic gastrointestinal dysfunction. China Medical Herald 2020;17:45-7.

31. Zhang $\mathrm{XW}$, Zhang $\mathrm{MZ}$, Weng $\mathrm{YN}$, et al. Intervention to gastrointestinal dysfunction and failure with therapy of relaxing bowels and purgation in critical patients. Journal of Beijing University of Traditional Chinese Medicine 2011;34:707-10.

32. Zhu HY. Treatment of postoperative gastrointestinal dysfunction with Tongfu Jiedu Prescription in critically ill patients. Guangzhou: Guangzhou University of Chinese Medicine, 2012.

33. Zhang R, Wang DD, Wang XP, et al. Advances in treatment of septic gastrointestinal dysfunction with rhubarb root and rhizome. Journal of Emergency in Traditional Chinese Medicine 2019;28:1672-74.

(English Language Editor: J. Jones)

Cite this article as: Zhang L, Wu Q, Chen P, Xu Y, Zheng Y, Ma S, Zheng J. Effect of Tongfu traditional Chinese medicine preparation on patients with septic gastrointestinal dysfunction: a systematic review and meta-analysis. Ann Palliat Med 2021;10(12):12072-12085. doi: 10.21037/apm-21-2461 